\title{
Polyamide fabric coated with a DHA-loaded chitosan hydrogel for a cosmeto-textile application
}

\begin{abstract}
Cosmeto-textiles, which allow the administration of molecules when in contact with the skin, are increasingly being developed by cosmetic industries. We have designed an innovative approach for cosmeto-textile products, based on the impregnation of textile fibers with chitosan hydrogels, which have been cross-linked with genipin and loaded with dihydroxyacetone (DHA), which is an active component that induces sunless tanning. DHA-loaded chitosan hydrogels have been prepared and characterized by means of cryogenic scanning electron microscopy (Cryo-SEM). The images showed that genipin cross-linking decreases the mesh distance of hydrogels. The release of DHA from these cross-linked genipin chitosan hydrogels has been studied by a dialysis membrane method. These DHA-loaded chitosan hydrogels have been incorporated to polyamide textiles by a simple padding technique. The presence of DHA on these textiles has been detected by hyperspectral imaging on a dark field high resolution optical microscope. Finally, the performance of fabrics as cosmeto-textiles, with a tanning effect, has been evaluated by skin-colorimetry, measured with an evaluation panel of 10 people. The results have demonstrated that DHA-loaded textiles produce a tanning effect on skin, and incorporation of DHA-loaded chitosan hydrogels into polyamide fabrics represents a friendly and appropriate strategy to obtain a cosmeto-textile with tanning effect.
\end{abstract}




\section{Keywords}

Cosmeto-textile, chitosan hydrogel, genipin, DHA, tanning effect

\section{Introduction}

Functionalized textiles and especially cosmeto-textiles are increasingly used for cosmetic applications [1]. A cosmeto-textile is a textile article containing a substance or a preparation intended to be durably released on the skin [2]. A cosmeto-textile allows the controlled release of molecules to the outer skin layers. The amount of compound transferred has to be sufficient to ensure that cosmetic benefits are possible [3]. There are several approaches to achieve specific textile functionalization; one of them is based on the impregnation of textile fibers with hydrogels. This technique presents two major advantages: the easy encapsulation of actives, of hydrophilic nature, into the hydrogel matrix by a simple pre-mixing step, and the easy impregnation of textiles with preformed hydrogels, by using a padder machine. Polyamide fabrics were selected for this study, for being a synthetic fiber with an increasing presence in medical material and healthcare/hygiene products with a lipophilic character [4].

Chitosan-based hydrogels have attracted considerable attention for biomedical applications [5-7] due to its low toxicity, good biocompatibility and biodegradability. Chitosan is a natural cationic polysaccharide composed of randomly distributed $\beta(1 \rightarrow 4)$ - 
linked N-acetylglucosamine and glucosamine. Among natural polymers, chitosan, an amino polysaccharide obtained by the deacetylation of chitin, has gained considerable attention from researchers worldwide. Chitosan is a biocompatible, biodegradable and high available cationic biopolymer which has already been used in textile finishing. This polymer has been used as textile finishing conferring antibacterial properties [8, 9], as adsorbent of anionic dyes $[10,11]$ or as shrink-resist agent in wool $[12,13]$, among other applications. This polymer has been selected because is highly available and it has been already used in textiles.

Hydrogels are hydrophilic three-dimensional (3D) cross-linked networks of highly hydrated molecules, which can swell in the presence of water, retaining a large amount of aqueous solution within their macromolecular structure. Chitosan hydrogels are crosslinked to provide stability to their 3D network. Several cross-linking reagents, such as glutaraldehyde, formaldehyde or epoxy compounds, have been used for cross-linking chitosan $[14,15]$. However, they present toxicity problems, which can be overcome by using genipin, a natural cross-linker reagent [16]. Genipin has been used to cross-link collagen and gelatin $[17,18]$, and also chitosan $[19,20]$. Genipin is a low-toxicity crosslinking agent, which is highly active on polymers with amino functional groups, such as chitosan, forming covalently cross-linked networks. Basically, the reaction mechanism goes first through a nucleophilic attack of the amino group of chitosan to a carboxymethyl group of genipin, leading to the formation of amide linkages. Subsequently, a slower second reaction is the nucleophilic substitution of the ester group of genipin by another amino group of chitosan forming a secondary amide link. This 
process is quite complex, since genipin can also react with itself forming active dimers and trimers [21]. Eventually, genipin form covalent bridges between two adjacent chitosan chains. Moreover, as polyamide fiber contains amino terminal groups, genipin can form covalent unions between these terminal groups and the amino groups of chitosan. Therefore, genipin can fix chitosan hydrogels on polyamide fibers, as well as cross-linking chitosan molecules. Thus, a stronger polymer network could be incorporated into the fabric.

The physical properties of chitosan hydrogels have enabled their use in drug delivery applications, in which the active ingredients or drugs are loaded in the hydrogel matrix. The porous structure of these hydrogels can be modulated by the polymer concentration and by the degree of cross-linking. This can modify the swelling extent of the network and therefore, have a direct impact on release of active ingredients [22].

Dihydroxyacetone, DHA, a three-carbon sugar, is the most-often used sunless tanning agent and appears commercially in skin creams, lotions, conditioners, aerosol foams, sprays, and fogs [23-25]. Commercial formulations typically contain from 1 to $10 \mathrm{wt} \%$ of this agent. DHA can react with free amino acids derived from peptides, in skin horny layer, via the Maillard reaction resulting in brown pigments known as melanoidins [26, 27]. Their formation can give the appearance of a natural skin tan without the risks associated with long-term exposure to sun radiation. In this context, textiles containing DHA may allow an increase in tanning when in contact with the skin. 
The aim of this study is designing an innovative approach for skin tanning based on polyamide fabrics coated with DHA-loaded chitosan hydrogels. To this end, we have first synthesized and characterized DHA-loaded chitosan hydrogels, by means of cryogenic electron microscopy and release studies. Then, these DHA-loaded chitosan hydrogels have been successfully incorporated to polyamide fabrics, and its efficacy as a cosmetotextile with tanning effect has been verified by an evaluation panel of 10 people.

Skin tanning by a cosmeto-textile coated with DHA-loaded chitosan hydrogels could be more comfortable and easy compared to other application methods. Possible commercial applications could be on elastic leggings, which are in homogeneous contact with skin.

\section{Experimental}

\section{Materials}

Chitosan medium molecular weight, (190-310 kDa, determined by viscosity measurements) with a deacetylation degree of $85 \%$ was purchased from Sigma-Aldrich in a powder form. Genipin (crystal-like powder, reagent grade) with an average molecular weight of $226 \mathrm{~g} / \mathrm{mol}$, and purity up $98 \%$ determined by HPLC, was supplied by Challenge Bioproducts Co. (Taiwan). Polyamide fabric 6.6 and DHA (dihydroxyacetone) were both supplied by AITEX (Spain). Glacial acetic acid was obtained from Panreac (Spain) with a purity of 99.5\%. Milli-Q deionized and filtered water and phosphate buffer solution (PBS, at $\mathrm{pH} 7.4$ or 5.5) were used for preparing solutions. Chitosan, genipin and DHA are shown in Fig. 1. 


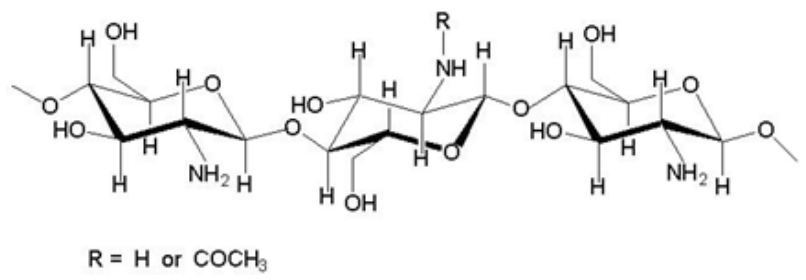

(a) Chitosan

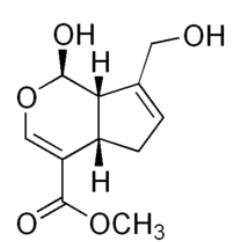

(b) Genipin<smiles>O=C(CO)CO</smiles>

(c) Dihydroxyacetone

Fig. 1. Schemes showing molecular structures of (a) Chitosan; (b) Genipin; and (c) DHA.

\section{Preparation of DHA-loaded chitosan hydrogels}

Chitosan solution at $1 \mathrm{wt} \%$ was prepared by dissolving chitosan powder in $1 \mathrm{vol} \%$ acetic acid by stirring overnight (magnetic stirring). The chitosan solution was then filtered, in order to remove chitosan insoluble impurities. Fresh genipin solutions were prepared by dissolving genipin powder in phosphate buffer at $\mathrm{pH} 7.4$ to produce solutions with genipin loadings in the range of $0.01-0.5 \mathrm{wt} \%$. This range of genipin concentrations was selected because it allowed cross-linking in a few hours of reaction time at $25^{\circ} \mathrm{C}$, as described in a previous publication [28]. Cross-linking reactions of chitosan with genipin occurs after mixing both solutions at 1:1 (w/w) ratio. DHA was added to the resulting pre-hydrogel solution in $3 \mathrm{wt} \%$. The molecular structure of cross-linked chitosan is illustrated in the scheme shown in Fig. 2. 


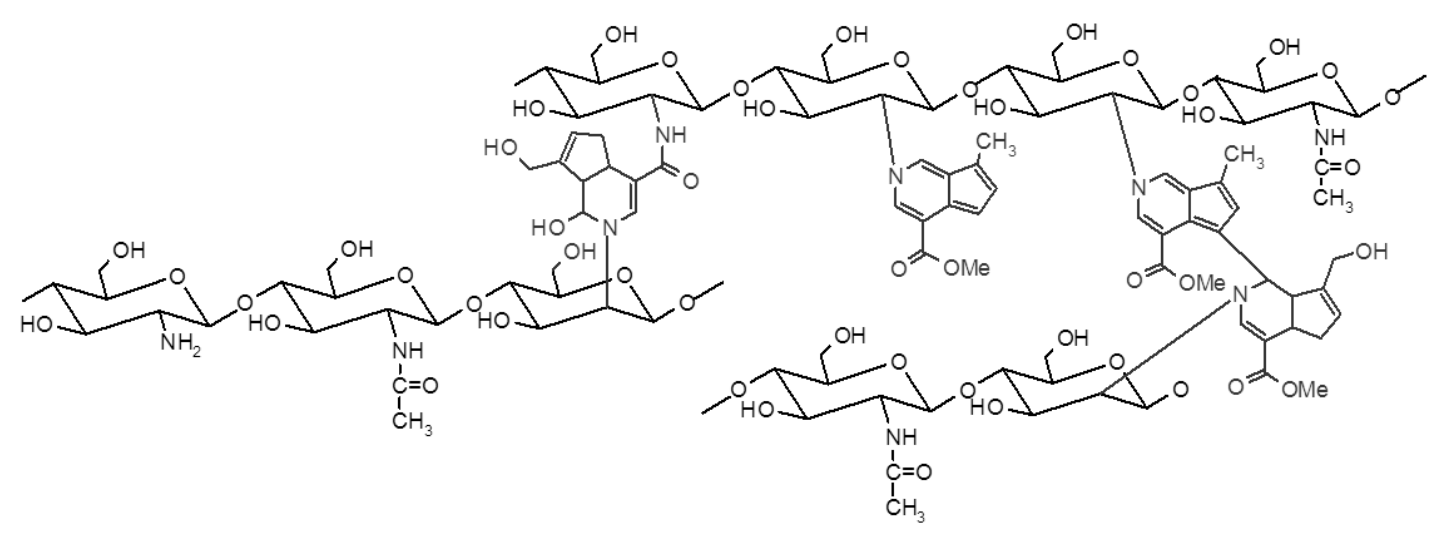

Fig. 2. Example of the molecular structure of three chitosan polymer chains, cross-linked by genipin bridges, which might consist of either one single genipin monomer or short gepinin oligomers (Adapted from [29]).

\section{Cryogenic Scanning Electron Microscopy.}

Cryogenic Scanning Electron Microscopy (cryo-SEM) was performed using a Hitachi S3500N scanning electron microscope (Hitachi, Japan) equipped with a cryo-chamber Quorum PP3000T. Prior to imaging, the hydrogel samples were rapidly frozen in liquid nitrogen and fractured inside a cryo chamber at $-130{ }^{\circ} \mathrm{C}$. After sublimation for $60 \mathrm{~s}$ at $-100{ }^{\circ} \mathrm{C}$ in vacuum, samples were sputtered with a thin platinum layer.

\section{DHA release studies.}


Diffusion experiments were carried out in a dissolution instrument (Hanson Vision Elite 8TM) set with automatic sampling (Hanson Research Autoplus Maximixer TM and MultifillTM), by using the dialysis membrane method. It consists of eight dissolution vessels with receptor solution immersed in a thermostated bath [31]. Dissolution testing is an extensively applied method which allows the release study without any kind of manipulation. Moreover, this in vitro method has been used because it is a simple method for comparing formulations and evaluating the release of DHA from hydrogels, despite the fact it does not represent in vivo conditions [32]. In the present case, a dialysis bag was used as sample holder, given the soft nature of hydrogels, even though such bags might cause a short delay in drug release.

DHA was incorporated in the pre-hydrogel matrix, namely the solution of all components before gelation, at $3 \mathrm{wt} \%$ concentration. An aliquot of each hydrogel ( $3 \mathrm{~g})$ was placed in a pre-swollen dialysis bag with a 12000-14000 Da molecular weight cutoff (Spectra/Por dialysis membrane; Spectrum Laboratories, Auckland, NZ), and immersed into $500 \mathrm{ml}$ of receptor PBS solution at pH 5.5 and temperature $32^{\circ} \mathrm{C}$ (scheme shown in Fig. 3). The membrane molecular weight cut off is appropriate to allow DHA release but preventing chitosan diffusion, which has a much larger molecular weight than DHA.

The dialysis bag was placed at the bottom of the vessel and the distance between the paddle and the sample was constant in all experiments. The receptor solution was PBS at $\mathrm{pH} 5.5$ at $32^{\circ} \mathrm{C}$. The stirring speed of the paddles in each dissolution vessel was $25 \mathrm{rpm}$. This slow stirring speed was considered appropriate, given the volume of the vessel (500 
$\mathrm{ml}$ ), and allowing a fast homogenization of the receptor solution. Samples were withdrawn at predetermined time intervals over an 8-hours period. Concentration of DHA was calculated by measuring the absorbance at $271 \mathrm{~nm}$. All absorbance determinations were carried out by using an Epoch Microplate Spectrophotometer (BioTek, USA), controlled with Gen5 Data Analysis software interface. All diffusion experiments were repeated at least twice. Although the results obtained with the dialysis bag method are not comparable to those resulting in physiological conditions, the setup is useful for comparative studies between formulations.

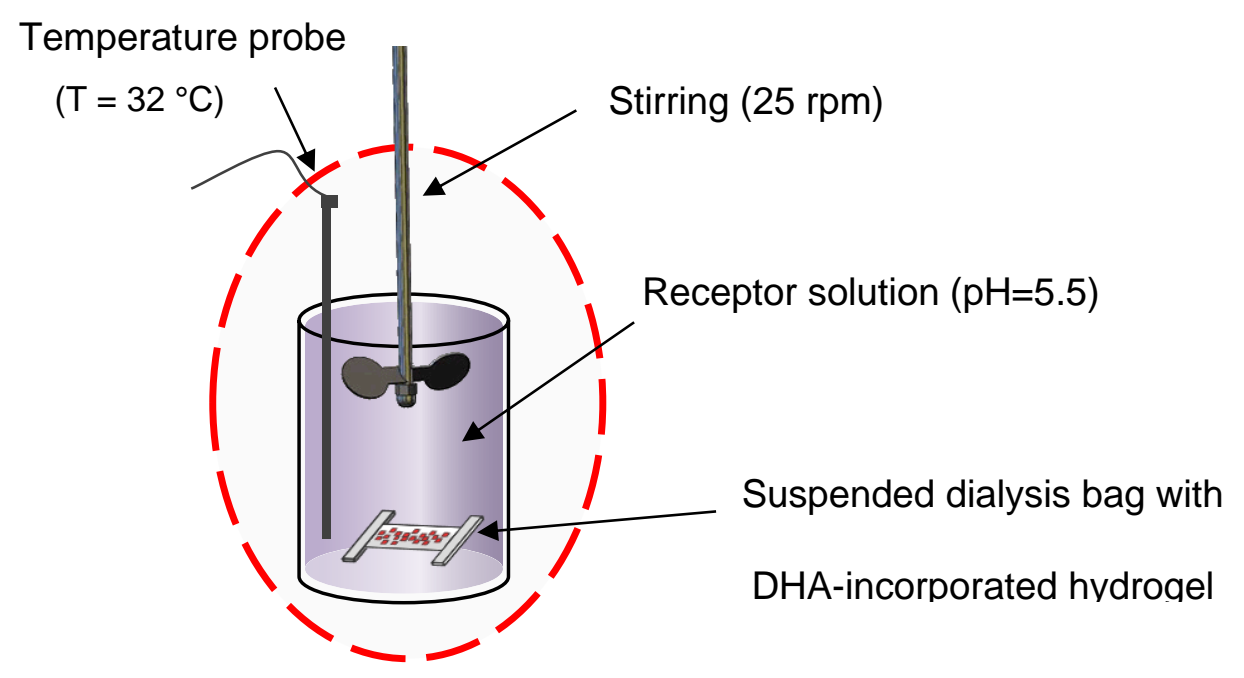

Fig. 3. Scheme describing the experimental setup of release essays.

To investigate the kinetics of DHA release from hydrogels, Higuchi model was used for fitting the results [33]. Higuchi proposed in 1961 the first example of a mathematical model aimed to describe drug release from a matrix system. 


\section{Hydrogel application to polyamide fabrics. Nano-scale optical and spectral characterization.}

Chitosan hydrogels cross-linked with genipin were applied on polyamide fabrics by the padding technique. Fabrics were immersed in hydrogel solutions and run three times through squeeze rolls with an HVF laboratory padder (Mathis, Switzerland), at $3 \mathrm{~m} / \mathrm{min}$ padding speed and 3 bar of squeeze roll pressure. Finally, the fabrics impregnated with hydrogels were left to dry at room temperature. Chitosan hydrogels become attached to polyamide fibers. Polyamide contains amino terminal groups, and consequently, genipin can form covalent bridges between chitosan and polyamide fibers.

In order to verify that DHA was successfully incorporated in the textile, polyamide fabrics coated with a hydrogel with $1 \%$ chitosan, $0.1 \%$ genipin and 3\% DHA, were characterized by means of hyperspectral imaging and mapping of this compound by using an optical microscope with enhanced dark field (EDF) and a powerful illumination source (Cytoviva, Auburn, AL, USA). The hyperspectral image was obtained with a concentric imaging visible and near-infrared (VNIR) spectrophotometer $(400-1000 \mathrm{~nm})$ with integrated CCD camera, and is controlled by ENVI 4.8 software for hyperspectral image analysis. A reference spectral library of the active was created prior to analysis of experimental samples, and such samples were compared to that reference, in order to examine the presence of DHA. 


\section{Efficacy as cosmeto-textiles. Tanning studies.}

DHA skin tanning effect, on a panel of 10 people, was assessed visually as well as quantified with a skin-colorimeter (CL400, Courage \& Khazaha), which characterizes the surface color of an object from the colorimetric measures $\mathrm{L}^{*}, \mathrm{a}^{*}$, and $\mathrm{b}^{*}$, which result from the CIE tristimulus values $\mathrm{X}, \mathrm{Y}, \mathrm{Z}$ [34]. $\mathrm{L}^{*}$ is referred to as the metric lightness and measures how light or dark a color is on an achromatic basis (relative to a matching shade of gray), ranging from $L^{*}=0$ for black to $L^{*}=100$ for white. Hue is a color appearance parameter defined in terms of the two chromaticity coordinates $a^{*}\left(a^{*}>0\right.$ indicates redness) and $b^{*}\left(b^{*}>0\right.$ indicating yellowness). The desire to better characterize the color as well as the lightness/darkness of the treated skin in terms of factors more relevant to human skin led to adoption of the Individual Typologic Angle $\left(\mathrm{ITA}^{\mathrm{O}}\right)$, proposed by Chardon et al. [35] as a quantitative and objective means of describing the intensity of skin pigmentation. The ITA values reported are the average measurements of 10 people. Then, the measured $\mathrm{L}^{*}$ and $\mathrm{b}^{*}$ were converted to the corresponding $\operatorname{ITA}^{\circ}$, expressed in degrees as $\operatorname{ITA}^{\circ}=57.3$ ArcTangent $\left(\left(\mathrm{L}^{*}-50\right) / \mathrm{b}^{*}\right)$. The change in $\operatorname{ITA}^{\circ}$ as a result of treatment, $\Delta \mathrm{ITA}^{\circ}$, is the quantity reported and was taken in such a way that larger values signify an increase in tan intensity.

Textiles coated with hydrogels with $1 \%$ chitosan, $0.1 \%$ genipin and 3\% DHA were applied on the skin (on the forearm), and after 4 days, tanning was determined by the 
change in ITA $^{\circ}$ angle values (Individual Typological Angle) as compared with control skin regions, which were exposed to the same textile but in absence of tanning agent.

\section{Results and discussion}

\section{Preparation of DHA-loaded chitosan hydrogels}

Hydrogels were formed by cross-linking chitosan with different genipin concentrations, in the range $0.01-0.5 \mathrm{wt} \%$ in weight, at $25^{\circ} \mathrm{C}$. In the case of DHA loaded hydrogels, 3 wt\%, respect to hydrogel total weight, was incorporated. Its gelation process was followed over time (Fig. 4). Initially, both non-loaded and DHA-loaded hydrogels were liquid and optically clear. For non-loaded hydrogels, after $4 \mathrm{~h}$ of its preparation, gelation was achieved for genipin concentrations of $0.1 \mathrm{wt} \%$ and higher. However, for DHAloaded hydrogels, gelation required higher genipin concentrations, achieved at 0.25 and $0.5 \mathrm{wt} \%$ genipin.

By increasing aging time to $24 \mathrm{~h}$, for non-loaded hydrogels, gelation is achieved at lower genipin concentrations, from $0.05 \mathrm{wt} \%$. It also should be noted that chitosan hydrogels become dark blue, in presence of genipin. This color change is attributed to formation of genipin derivatives produced by reactions of genipin with primary amines [21, 29, 30]. Therefore, color change is associated with cross-linking reactions.

In the case of DHA-loaded hydrogels, at the same aging time of $24 \mathrm{~h}$, gelation is only achieved at genipin concentrations of $0.1 \mathrm{wt} \%$ and higher. Consequently, the presence of 
DHA retards gelation of hydrogels, showing a slower gelation kinetics if compared to non-loaded DHA hydrogels at the same genipin concentration. This is due to the existence of DHA-chitosan interactions, which reduces cross-linking, as described in a previous paper [36].

WITHOUT ACTIVE

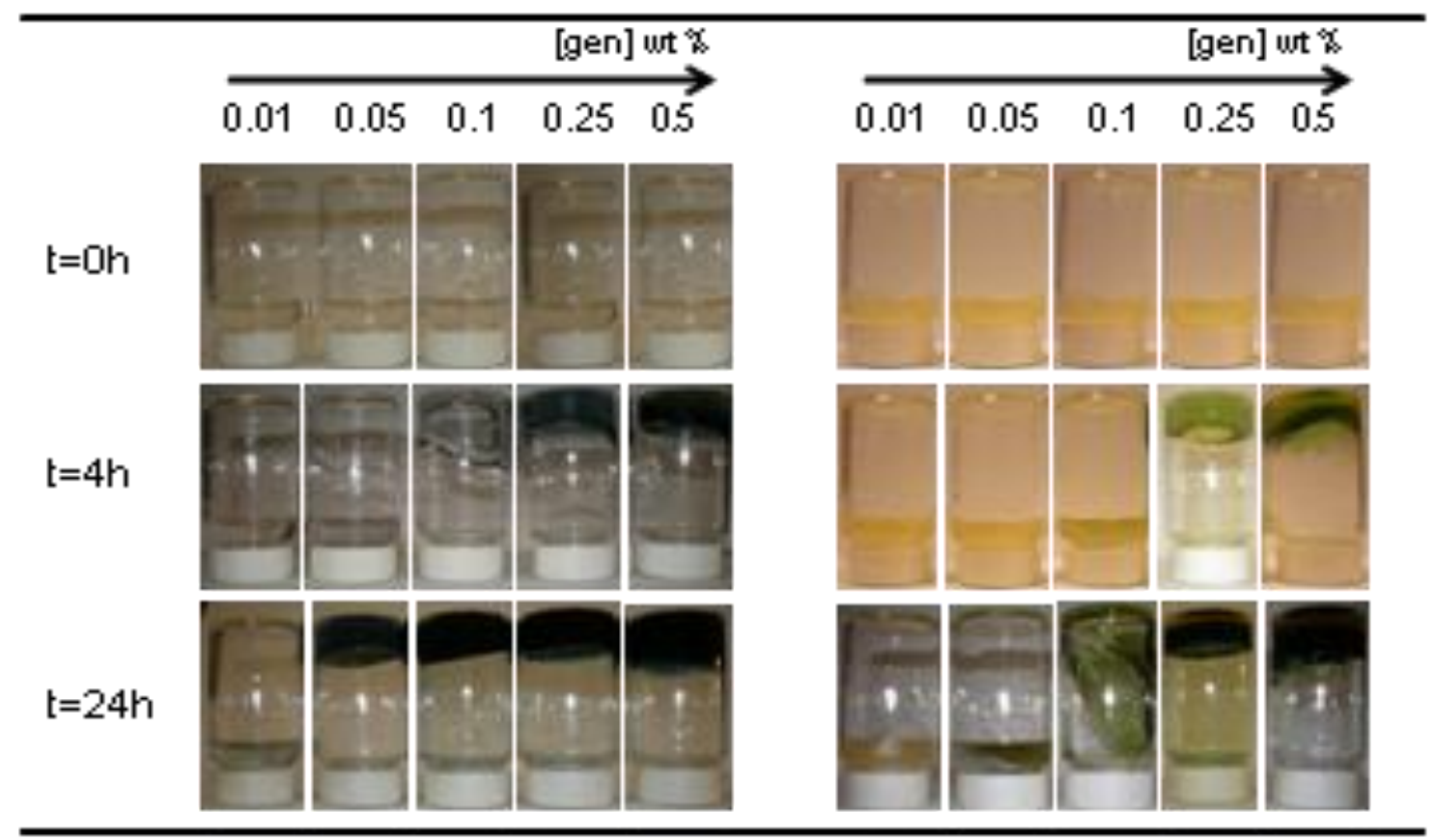

Fig. 4. Appearance of non-loaded and DHA-loaded chitosan hydrogels at different genipin concentrations, just after their preparation, and $4 \mathrm{~h}$ and $24 \mathrm{~h}$ after their preparation. Vials have been turned upside down, to show the slow flowing of viscous samples.

A Maillard reaction would be expected to occur between the carbonyl group of DHA and free amino group of chitosan, losing a mole of water and forming an $\mathrm{N}$-substituted glycosylamine, similar to that occurred between skin aminoacids and DHA. Dehydration 
leads to the formation of a Schiff base and then a rearrangement reaction produces a Heyns product [27].
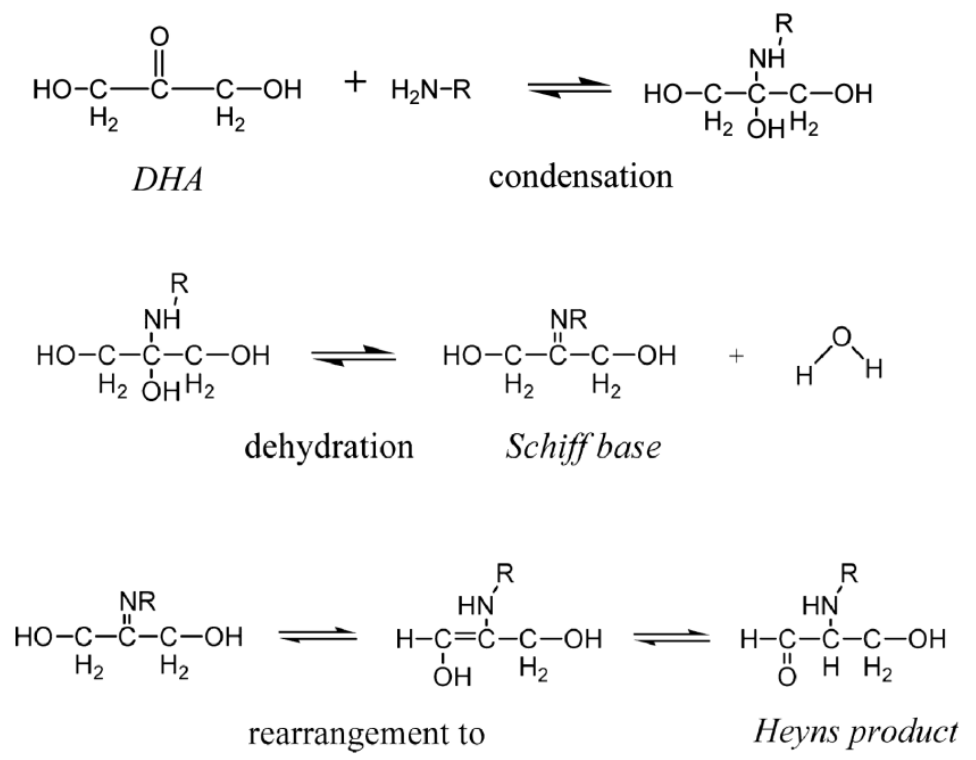

Fig.5. Maillard reaction between the DHA carbonyl group and a free amino group. The initial step is a condensation, and a Schiff base is formed after dehydration. Finally, a Heyns product is released. (Reproduced from Ref. [27], with permission from the American Chemical Society).

When chitosan is cross-linked with genipin the mechanism goes, firstly, through a nucleophilic attack of the amine group of chitosan to a carboxymethyl group of genipin, leading to the formation of amide linkages. Subsequently, a nucleophilic substitution of the ester group of genipin by the amine group of chitosan takes place, forming a secondary amide link [21]. However, when DHA is present in the system, the nucleophilic attack of the amine group of chitosan could be produced over the carbonyl 
group of DHA, producing such an active-polymer interaction which would extend the gelation time in DHA loaded hydrogels.

\section{Morphology of hydrogels by Cryo-SEM observation}

Structure and morphology of synthesized hydrogels were examined by using a cryogenic scanning electron microscope, cryo-SEM [37]. Both the effects of the cross-linking density and presence of DHA were investigated. Figure 6a-c shows the effect of the amount of cross-linker added on the structure of the hydrogels. Hydrogels without crosslinker form a laminated structure (Fig. 6a). By increasing genipin concentration to 0.1 wt\% (Fig. 6b) and to 1 wt\% (Fig. 6c), the mesh distance decreases. On the other hand, Figure $6 \mathrm{~d}$ shows the Cryo-SEM image of hydrogels at $0.1 \mathrm{wt} \%$ genipin concentration with 3 wt\% loaded DHA. This sample shows a larger mesh distance (Fig. 6d) in comparison with the same hydrogel composition but in absence of DHA (Fig. 6b).
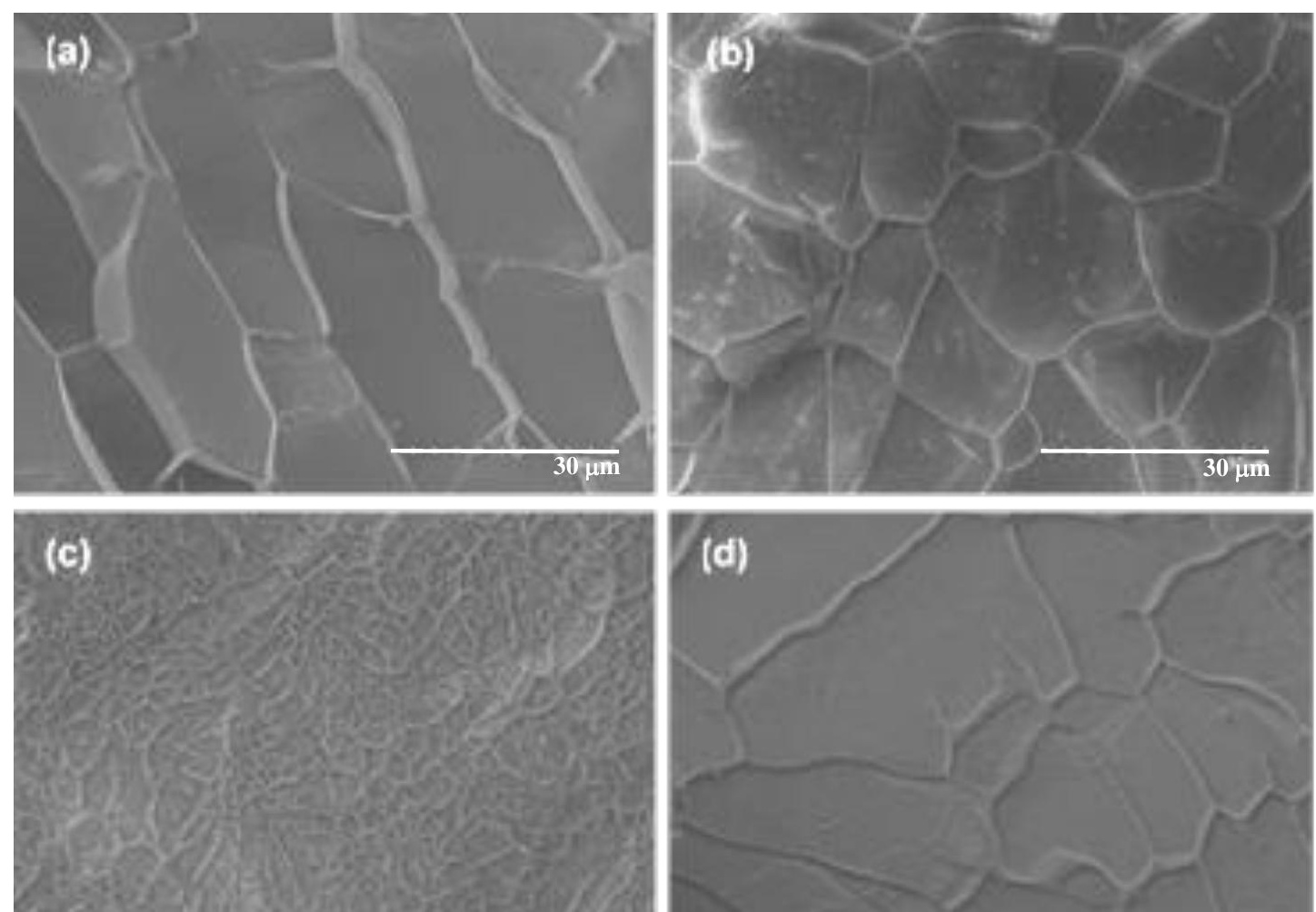
Fig. 6. Cryo-SEM images of chitosan hydrogels without genipin (a), with 0.1 wt\% genipin concentration (b) and with $1 \mathrm{wt} \%$ genipin concentration (c). These samples did not contain DHA. In contrast, (d) shows the Cryo-SEM image at $0.1 \mathrm{wt} \%$ genipin concentration in presence of $3 \mathrm{wt} \%$ loaded DHA.

Interpretation of cryo-SEM images should be cautious, since artifacts could be confused with real morphological features. However, in the present images one can clearly observe that the characteristic distance between structures becomes shorter as genipin concentration is increased (Fig 6a, 6b and 6c). Certainly, this is an indication of a reduction of mesh distance with genipin concentration. Moreover, the larger structures observed in the DHA-loaded chitosan hydrogel (Fig. 6d) are in very good agreement with the slower gelation velocity shown by DHA-loaded chitosan hydrogels (Fig. 4). This is due to a lower cross-linking degree, caused by weak non-covalent interactions between DHA and chitosan, as reported before [36], which was attributed to hydrogen bonds.

\section{DHA release studies}


DHA release from chitosan hydrogels was studied by the dialysis membrane method, as described in detail in the experimental section. Figure 7A shows the cumulative amount of DHA measured in the compartment of PBS receptor solution, as a function of time. Various donor systems, with 3wt\% DHA, were compared: (a) DHA released from an aqueous solution; (b) from a non-cross-linked chitosan hydrogel, (c) from cross-linked chitosan hydrogels with genipin cross-linker concentration of $0.1 \mathrm{wt} \%$ and (d) with 0.5 wt\% genipin concentration.

A marked reduction in release velocity was observed when genipin was added for crosslinked chitosan, in comparison with DHA in aqueous solution or non-cross-linked chitosan hydrogels. Moreover, release rate velocity decreases with cross-linker concentration. This slower DHA diffusion from cross-linked chitosan hydrogels could be attributed to a denser and more viscous network. This reduction in diffusion velocity was expected and it has also been observed elsewhere for cross-linked hydrogels [31]. Interestingly, the two samples without genipin (aqueous solution and uncross-linked hydrogel) produced almost identical results. In conclusion, the results confirm that DHA is not retained in the hydrogel and their release velocity can be controlled by tuning the degree of cross-linking.

(A)

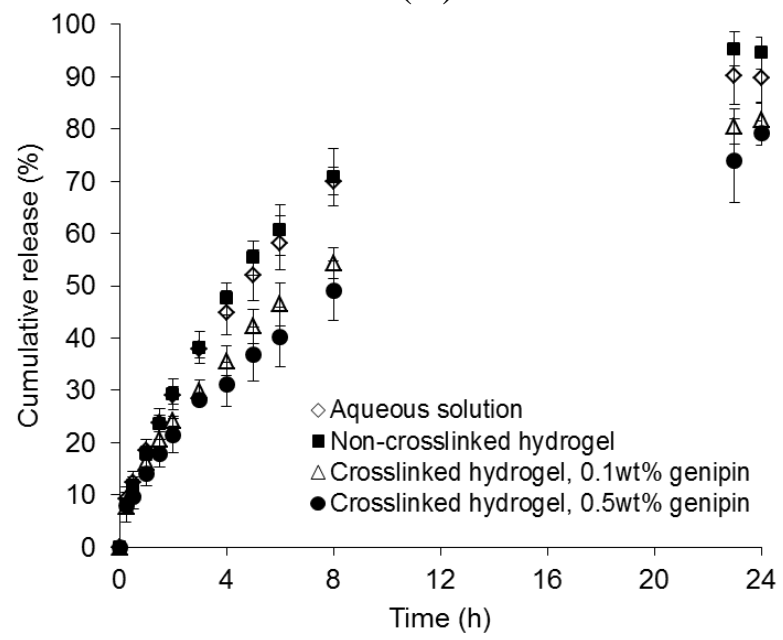

(B)

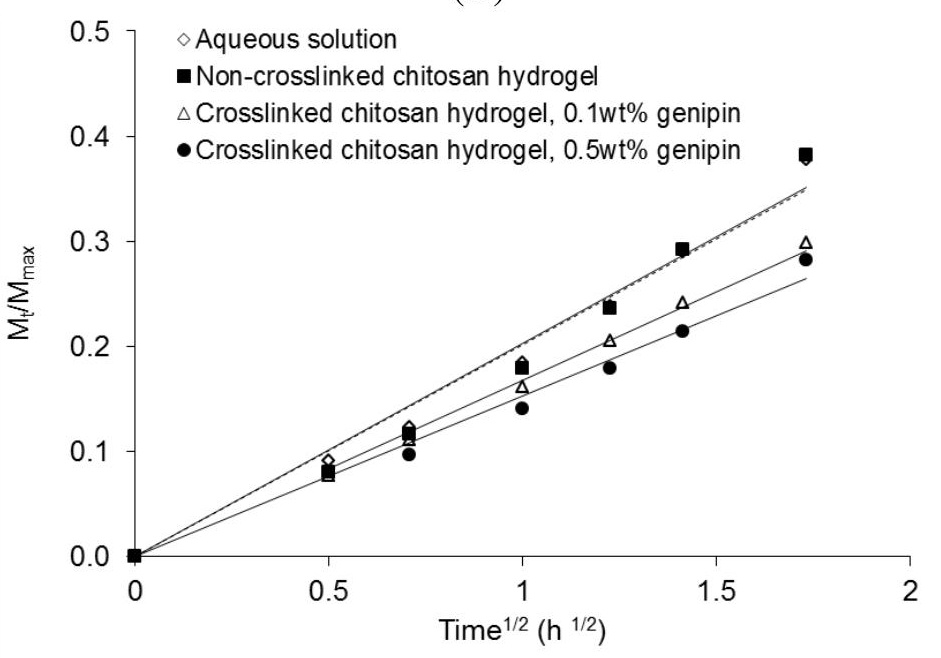


Fig. 7. DHA release studies from an aqueous solution, chitosan solution, and hydrogels cross-linked with different genipin concentrations of 0.1 and $0.5 \mathrm{wt} \%$. (A) Cumulative release as a function of time. The line is a visual guide, and the error bars indicate the standard deviation; (B) Relative released amount, $\mathrm{M}_{\mathrm{t}} / \mathrm{M}_{\max }$, showing the released amount $M_{t}$ relative to the total amount $M_{\max }$ at short times $(t \leq 3 h)$. The lines indicate fits to Higuchi equation. Release of non-crosslinked hydrogel cannot be distinguished from release of aqueous solution and most points overlap.

With regards to the mechanism of DHA release, plotting the amounts of tanning agent released against the square root of time, straight lines were obtained with a rather good fit at short times (Fig. 7B). These linear plots might indicate that DHA release in these systems is diffusion controlled, in accordance with the equation developed by Higuchi [33] for diffusion controlled release of active ingredients from semi-solid matrices. Nevertheless, it should be noted that DHA interacts with the amino groups, forming an intermediate Schiff base, as described in Figure 5. Therefore, release of DHA has a very complex mechanism, which cannot be explained by Higuchi equation based on a simple diffusion process. 
However, the fact that a rather good linear fit is obtained by plotting the amounts of DHA released, versus the square root of time, allows us to use this fit for comparing the different systems studied. The Higuchi release kinetic parameter was calculated, and it is shown in Table 1.

Table 1. Results of fitting Higuchi equation $\left(\mathrm{M}_{t} / \mathrm{M}_{\max }=\mathrm{k}_{\mathrm{h}} \mathrm{t}^{1 / 2}\right)$ to the release of DHA from various systems at short times ( $\mathrm{t} \leq 3$ hours). The Higuchi kinetic parameter and the correlation factor $\left(\mathrm{R}^{2}\right)$ are shown.

\begin{tabular}{lcc}
\hline \multicolumn{1}{c}{ System } & $\begin{array}{c}\text { Higuchi release } \\
\text { parameter }\left(\mathbf{k}_{\mathbf{h}}\right)\end{array}$ & $\mathbf{R}^{\mathbf{2}}$ \\
\hline Aqueous solution & 0.20 & 0.983 \\
Non cross-linked chitosan hydrogel & 0.20 & 0.973 \\
$\begin{array}{l}\text { Cross-linked chitosan hydrogel } \\
\text { ([GEN]=0.1 wt\%) }\end{array}$ & 0.17 & 0.993 \\
$\begin{array}{l}\text { Cross-linked chitosan hydrogel } \\
\text { ([GEN]=0.5 wt\%) }\end{array}$ & 0.15 & 0.987 \\
\hline
\end{tabular}

The results of Higuchi kinetic parameter clearly show that cross-linking slows down release kinetics. The values of this parameter decrease from 0.20 to 0.15 , by increasing genipin from zero to $0.5 \mathrm{wt} \%$. These values can be compared to that of DHA in simple aqueous solution in absence of both chitosan and genipin, which produces exactly the same velocity of release, with $\mathrm{k}_{\mathrm{h}}=0.20$. 


\section{Detection of DHA in textiles.}

DHA-loaded chitosan hydrogels were applied on textiles by the padding technique. In order to understand if this active component was homogenously incorporated to the textile, treated textiles were examined using the CytoViva VNIR hyperspectral imaging system coupled to high resolution darkfield microscopy. The tanning agent has a significantly different refractive index from the textile, which leads to greater scattering of light in the sections containing it, and hence, its easy detection. Figure 8a illustrates a spectral scan of DHA in powder form at 10X. From this image, different pixels were selected to build a spectral library (shown in Figure 8b), which is used to confirm the presence of DHA. Figure 8c corresponds to a spectral scan of the textile fibers at 10X. Finally, Figure 8d shows the mapping of DHA. Pixels in red map the presence of DHA spectra, illustrating that DHA was successfully and homogeneously incorporated in the textile.
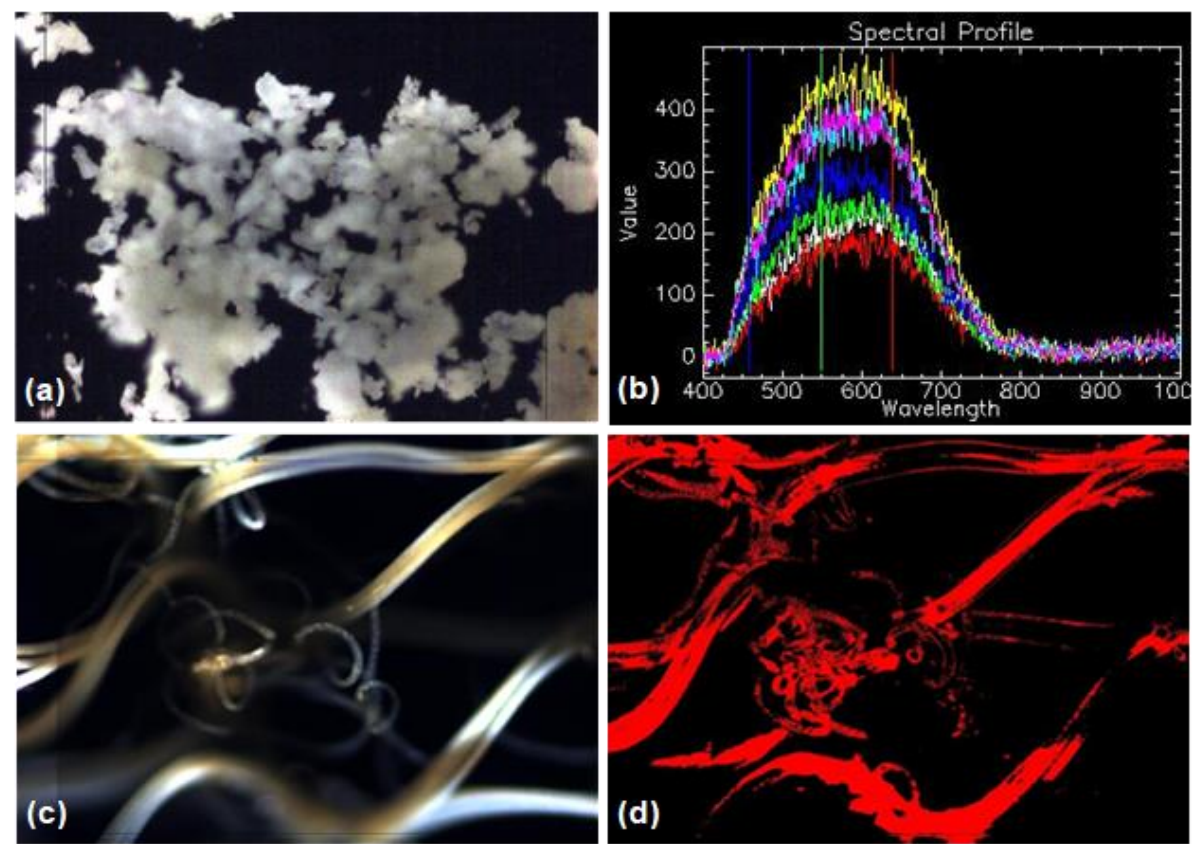
Fig. 8. (a) Spectral scan of DHA, (b) DHA spectral library, (c) spectral scan of polyamide textile, and (d) mapping of DHA in the polyamide textile.

\section{Efficacy as a cosmeto-textiles. Tanning studies.}

The cosmeto-textile tanning efficacy was evaluated by assessing change in skin color. Textiles coated with DHA-loaded chitosan hydrogels were applied on the skin (forearm) of a 10 people panel, and both a qualitative observation and quantitative determination (by using a colorimeter) were assessed during a time period of 4 days. Regarding the qualitative observation, it was based on the opinion of the 10 panel people. After 4 days of the contact of the textile with the skin, 6 of the panellists considered that a tanning effect was produced. In this group of volunteers, 4 of them considered that the tanning was aesthetic, with no yellowness. Much more precise evaluation was performed by quantitatively measuring the change of skin color, by using a colorimeter applied on the skin and comparing before and after the application of DHA-loaded fabrics. The results were compared to those of skin regions that were treated with the same hydrogel-coated textiles, but in absence of DHA (control regions). After $8 \mathrm{~h}$ and 4 days, ITA $^{\circ}$ values were obtained. In Figure 9 it is shown the evolution of ITA $^{\circ}$ values for the control region and the treated region as a function of time. The lower the $\operatorname{ITA}^{\circ}$ value obtained, the higher the tanning effect. After 4 days, a decrease of a $7.3 \%$ in the ITA $^{\circ}$ parameter for the treated 
region was observed and, hence, we could quantitative demonstrate that skin contact with the cosmeto-textile produces a tanning effect.

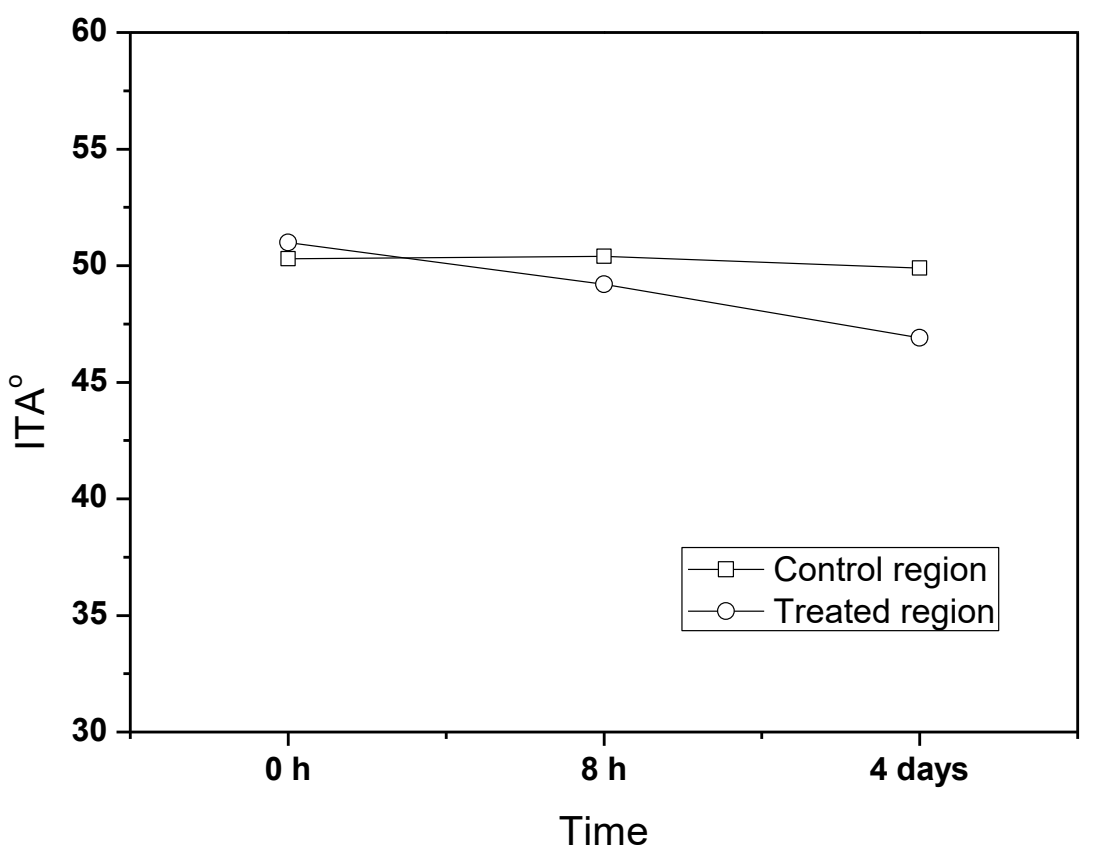

Fig. 9. Quantitative $\mathrm{ITA}^{\mathrm{o}}$ values, calculated from skin-colorimetry determinations, show the evolution for the control and treated regions as a function of time.

The tanning effect was also evaluated on a volunteer panelist wearing polyamide leggings for different times, shown in Figure S1, enclosed as supporting information. Leggings were treated with a pre-hydrogel solution (1\% chitosan, $0.1 \%$ genipin, $25 \%$ DHA) by the 
padding technique. The DHA concentration was increased to evaluate the tanning effect in a shorter period of time.

\section{Conclusions}

In this study, we have designed an innovative approach for tanning of the skin based on polyamide fabrics coated with DHA-loaded chitosan hydrogels. Dihydroxyacetone, DHA, has been successfully incorporated in chitosan hydrogels, and characterized by means of Cryo-SEM. A reduction of hydrogel mesh distance was detected by increasing genipin concentration. Moreover, the release velocity of DHA from hydrogel can be controlled by tuning the degree of cross-linking. These DHA-loaded chitosan hydrogels were applied into polyamide fabrics, by using a simple padding technique and it was confirmed that DHA was successfully and homogeneously incorporated in the textile. The efficacy of this cosmeto-textile as a tanning product when in contact with the skin has been measured by skin-colorimetry. The results clearly proved that DHA-loaded textiles had a tanning effect. Thus, we can conclude that the incorporation of DHAloaded chitosan hydrogels into polyamide fabrics represents a friendly and appropriate strategy to obtain a cosmeto-textile with tanning effect. 


\section{Acknowledgments}

The authors acknowledge financial support from CDTI (INNPACTO program), grant IPT-300000-2010-0026. The optical microscope observations have been performed by the Nanostructured Liquid Characterization Unit, located at the Institute of Advanced Chemistry of Catalonia (IQAC), belonging to the Spanish National Research Council (CSIC) and affiliated to the NANBIOSIS ICTS of the Biomedical Networking Center (CIBER-BBN).

\section{References}

[1] Petrusic S and Koncar V. Controlled release of active agents from microcapsules embedded in textile structures. In: Koncar V (ed) Smart Textiles and Their Applications. Cambridge: Woodhead Publishing, 2016, pp. 1-14.

[2] Persico P and Carfagna C. Cosmeto-Textiles: State of the Art and Future Perspectives. Adv. Sci. Tech., 2013; 80, 39-46.

[3] Paul R. Functional finishes for textiles: an overview. In: Roshan P (ed) Functional Finishes for Textiles. Cambridge: Woodhead Publishing, 2015, pp. 1-14.

[4] Volkmar TB. Physiological comfort of biofunctional textiles. In: Hipler UC, Elsner P (eds.) Biofunctional Text. Ski. Curr. Probl. Dermatol, Basel: Karger, 2006, pp. 5156.

[5] Berger J, Reist M, Mayer JM et al. Structure and interactions in covalently and ionically crosslinked chitosan hydrogels for biomedical applications. Eur J Pharm Biopharm 2004; 57:19-34.

[6] Ahmadi F, Oveisi Z, Samani SM et al. Chitosan based hydrogels: characteristics and pharmaceutical applications. Res Pharm Sci 2015; 10: 1-16.

[7] Cardoso MJ, Costa RR and Mano JF. Marine Origin Polysaccharides in Drug Delivery Systems. Mar Drugs 2016;14: 1-27. 
[8] Lim SH and Hudson SM. Review of chitosan and its derivatives as antimicrobial agents and their uses as textile chemicals. J Macromol Sci Polymer Rev 2003; 43(2) 223-269.

[9] Gao Y and Cranston R. Recent advances in antimicrobial treatments of textiles.Text Res J 2008; 78 (1) 60-72.

[10] Rinaudo M. Chitin and chitosan: properties and applications. Prog Polym Sci 2006; 31: 603-632.

[11] Ravi Kumar MNV. A review of chitin and chitosan applications. React Funct Polym 2000; 46:1-27.

[12] Julià MR, Pascual E and Erra, P. Influence of the molecular mass of chitosan on shrink-resistance and dyeing properties of chitosan-treated wool, J Soc Dyers Colour 2000; 116: 62-67.

[13] Vílchez S, Manich AM, Jovancic P et al. Chitosan contribution on wool treatment with enzyme, Carbohyd Polym 2008; 71:515-523.

[14]Chen S, Liu M, Jin S at al. Synthesis and Swelling Properties of pH-Sensitive Hydrogels Based on Chitosan and Poly (methacrylic Acid) Semi-interpenetrating Polymer Network. J Appl Pol Sci 2005; 98:1720-1726.

[15] Yin L, Fei L, Cui F, et al. Superporous hydrogels containing poly(acrylic acid-coacrylamide)/O-carboxymethyl chitosan interpenetrating polymer networks, Biomaterials 2007; 1258-1266.

[16] Sung HW, Huang RN, Huang LLH et al. In vitro evaluation of cytotoxicity of a naturally occurring cross-linking reagent for biological tissue fixation. J Biomater Sci Polym Ed 1999; 10: 63-78.

[17]Delgado LM, Fuller K and Zeugolis DI. Collagen Cross-linking: Biophysical, Biochemical and Biological Response Analysis. Tissue Eng Part A 2017; 23:10641077.

[18]Oryan A, Kamali A, Moshiri A et al. Chemical crosslinking of biopolymeric scaffolds:Current knowledge and future directions of crosslinked engineered bone scaffolds. Int J Biol Macrom 2018; 107:678-688.

[19]Moura MJ. Rheological study of genipin in cross-linked chitosan hydrogels. Biomacromolecules 2007; 8:3823-3829.

[20]Muzzarelli RAA. Genipin-cross-linked chitosan hydrogels as biomedical and pharmaceutical aids. Carbohydr Polym 2009; 77:1-9. 
[21] Butler MF, Ng YF and Pudney PDA. Mechanism and Kinetics of the Crosslinking Reaction between Biopolymers Containing Primary Amine Groups and Genipin. $J$ Polym Sci Part A: Polym Chem 2003; 41: 3941-3953

[22] Hoare TR and Kohane DS. Hydrogels in drug delivery: Progress and challenges. Polymer 2008; 49: 1993-2007.

[23] Dicianna V. Self-tanning composition in sheeted substrate. Patent 7198780 B2, USA, 2007.

[24] Brillouet AS. Self-tanning composition. Patent 20090226386A1, USA, 2009.

[25] Vielhaber G, Schaper K and Herrmann M. Use of glycosylated flavonones for the browning of skin or hair. Patent 20080305054, USA, 2008.

[26] Lloyd RV, Fong AJ and Sayre RM. In Vivo formation of Maillard reaction free radicals in mouse skin. J Invest Dermatol 2001; 117:740-742.

[27] Carnali JO, Madison SA, Shah P, et al. Structure / Property Relationship for Ethylenediamine Derivatives as Aids in Sunless Tanning. Ind Eng Chem Res 2012; 51:15573-15581.

[28] Vílchez S, Samitier V, Porras M, et al. Chitosan Hydrogels Covalently Cross-linked with a Natural Reagent. Tens Surf Deter 2009; 46:13-17.

[29] Mi FL, Sung, H W and Shyu, SS. Synthesis and Characterization of a Novel Chitosan-Based Network Prepared Using Naturally Occurring Cross-linker. J Polym Sci Part A: Polym Chem 2000;15:2804-2814.

[30] Mi FL, Sung, H W and Shyu, SS. Drug release from chitosan-alginate complex beads reinforced by a naturally occurring cross-linking agent. Carbohydr Polym 2002; 48:61-72.

[31]Roig-Roig F, Solans C, Esquena J et al. Preparation, Characterization and Release Properties of Hydrogels Based on Hyaluronan for Pharmaceutical and Biomedical Use. J Appl Pol Sci 2013; 1377-1382.

[32] Son Y and McConville JT. Development of a standardized dissolution test method for inhaled pharmaceutical formulations. Int J Pharm 2009; 382:15-22.

[33] Higuchi T. Mechanism of sustained-action medication. Theoretical analysis of release of solid drugs dispersed in solid matrices. J Pharm Sc 1963; 52: 1145-1149.

[34] Vijayalekshmi SRP and Kamala C. Basic aspects and applications of tristimulus calorimetry. Talanta 1996; 43:1187-1206. 
[35] Chardon C, Cretois A and Hourseau I. Skin color typology and suntanning pathways. Int J Cosmet Sci 1991; 13: 191-208.

[36] Solé I, Vílchez S, Miras J, Montanyà N et al. DHA and L-carnitine loaded chitosan hydrogels as delivery systems for topical applications. Colloids Surfaces A 2017; 525 85-92.

[37] Yoshinobu ISM, Morita M andHiguchi M. Morphological Study of Hydrogels of Cellulosic Super Water Absorbents by CRYO-SEM Observation. J Appl Polym Sci 1994; 53: 1203-1209. 